\title{
IMPACT OF THE HIPPARCOS DATA ON THE ASTROMETRIC REDUCTION OF THE OUTER PLANETS
}

\author{
A. FIENGA \\ Bureau des Longitudes, 77, Av. Denfert-Rochereau 75014 Paris, France
}

We investigated the improvement of photographic astrometric observations of outer planets by use of Hipparcos Catalogue stars. The position of Jupiter on the plates was determined from measurements of the Galilean satellites, combined with ephemerides accurate to better than 10 mas. In a 6 contants model of photographic plates reduction, when images are near the center of the field, only offsets have to be computed, by considering the scale and orientation known. We applied this idea to the galilean satellites by using 137 photographic plates realised between 1967 and 1974 by D. Pascu, with the Mc Cormick 26 -inch refractor, Virginia, and its twin in the USNO, Washington DC. A study of the scale factors reveals an expected anisotropy of the scale factors. One possible hypothesis is that this effect is a consequence of the thermal expansions of the telescopic components. The use of Hipparcos data in the astrometric reduction allows to obtain an external error of around 40 mas on the determination of the observed absolute position of Jupiter. It is 5 times better than the classic computations using photographic or CCD reductions. Furthemore, with such results, badly-known effects can be easly estimated like the anisotropy of scale. At the end, important impacts on the fit of planetary ephemerides to the visual observations are expected.

\section{A PRELIMINARY STUDY ON THE IMPROVEMENT OF PROPER MOTIONS FOR HIPPARCOS STARS BY USING PHOTOGRAPHIC PLATES}

\author{
W. JIN, Z. TANG, J. LI, S. WANG \\ Shanghai Observatory, Academia Sinica, 80 Nandan Road, Shanghai 200030, China
}

The 3435 photographic plates, which were obtained by using the $40 \mathrm{~cm}$ astrograph at Zô-Sè station of Shanghai Observatory during 1901-1993, have been accumulated for observing open clusters, extragalatic nebulas, radio stars and variable stars etc. These plates cover 764 unoverlapping regions $\left(2^{\circ} .3 * 1^{\circ} .7\right)$ on the sky. Most of the plates distribute along the ecliptic, about 10000 Hipparcos stars, 27000 PPM stars and 500 FK5 stars are included in these regions. Among these plates, there are 2718 plates having two observing epochs, and they cover 359 different regions; there are 142 and 92 regions where the intervals of two epochs are about 50 and 70 years, and the numbers of plates in these regions are about 1494 and 1023 respectively. About 372 plates were photographed before 1949, which play an important action for improvement of proper motions of Hipparcos stars.

Four methods used to improve the proper motions for Hipparcos stars were designed. If the 2718 plates mentioned above having two epochs were used for improvement of the proper motions of Hipparcos stars, the stellar number of $299,1378,377$ and 1664 corresponding to the four methods will be obtained with final precision 0.7 mas/yr. If supplementary observations are made in 1997, the stellar number of 494,2431, 1534 and 2899 will be obtained with the same precision. 